\title{
Triple Differential Cross Sections for electron-impact ionization of methane at intermediate energy
}

DOI:

$10.1063 / 1.5097670$

\section{Document Version}

Accepted author manuscript

Link to publication record in Manchester Research Explorer

\section{Citation for published version (APA):}

Ali, E., Granados, C., Sakaamini, A., Harvey, M., Ancarani, L. U., Murray, A., Dogan, M., Ning, C., Colgan, J., \& Madison, D. (2019). Triple Differential Cross Sections for electron-impact ionization of methane at intermediate energy. Journal of Chemical Physics. https://doi.org/10.1063/1.5097670

\section{Published in:}

Journal of Chemical Physics

\section{Citing this paper}

Please note that where the full-text provided on Manchester Research Explorer is the Author Accepted Manuscript or Proof version this may differ from the final Published version. If citing, it is advised that you check and use the publisher's definitive version.

\section{General rights}

Copyright and moral rights for the publications made accessible in the Research Explorer are retained by the authors and/or other copyright owners and it is a condition of accessing publications that users recognise and abide by the legal requirements associated with these rights.

\section{Takedown policy}

If you believe that this document breaches copyright please refer to the University of Manchester's Takedown Procedures [http://man.ac.uk/04Y6Bo] or contact uml.scholarlycommunications@manchester.ac.uk providing relevant details, so we can investigate your claim.

\section{OPEN ACCESS}


Triple Differential Cross Sections for electron-impact ionization of methane at intermediate energy

Esam $\mathrm{Ali}^{1}$, Carlos Granados ${ }^{2}$, Ahmad Sakaamini $^{3}$, Matthew Harvey ${ }^{3}$, Lorenzo Ugo Ancarani ${ }^{4}$, Andrew James Murray ${ }^{3 *}$ Mevlut Dogan ${ }^{5}$, Chuangang Ning ${ }^{6}$, James Colgan ${ }^{7}$ and Don Madison $^{1}$

\author{
AFFILIATIONS \\ ${ }^{1}$ Department of Physics, Missouri University of Science and Technology, Rolla MO, USA \\ ${ }^{2}$ Institut für Physik, Martin-Luther Universität Halle-Wittenberg, 06199 Halle(Saale), \\ Germany \\ ${ }^{3}$ Photon Science Institute, School of Physics and Astronomy, University of Manchester, \\ Oxford Road, Manchester M13 9PL, UK \\ ${ }^{4}$ Université de Lorraine, CNRS, LPCT, 57000 Metz, France \\ ${ }^{5}$ ecol Academy, Afyon Kocatepe Konutları, CK1, C3-030916 Afyon, Turkey \\ ${ }^{6}$ Department of Physics, State Key Laboratory of Low-Dimensional Quantum Physics, \\ Tsinghua University, Beijing, China \\ ${ }^{7}$ Theoretical Division, Los Alamos National Laboratory, Los Alamos, NM, USA \\ *Email: Andrew.Murray@manchester.ac.uk
}

\begin{abstract}
We report an experimental and theoretical investigation of electron-impact single ionization of the HOMO $1 t_{2}$ and the NHOMO $2 a_{1}$ states of $\mathrm{CH}_{4}$ at an incident electron energy of 250 $\mathrm{eV}$. Triple differential cross sections measured in two different laboratories were compared with results calculated within the molecular 3-body distorted wave (M3DW) and generalized Sturmian function (GSF) theoretical models. For ionization of the $1 t_{2}$ state, the binary peak was observed to have a single maximum near the momentum transfer direction that evolved into a double peak for increasing projectile scattering angles, as has been seen for ionization of atomic p-states. A detailed investigation of this evolution was performed. As expected because of its s-type character, for ionization of the $2 a_{1}$ state only a single binary peak was observed. Overall, good agreement was found between experiment and theory.
\end{abstract}




\section{INTRODUCTION}

One of the most fundamental interactions in nature is that of electron-molecule collisions. Due to the complexity of this problem, theoretical calculations necessarily make a number of approximations, and the validity of these must be checked by comparing to experiment. For the case of electron-impact single ionization of molecules, the most stringent test of theory is provided by experimental measurements that determine all the energies and angular locations of the participants. Experiments of this type are labeled $(e, 2 e)$ measurements, and the associated cross sections are usually called triple differential cross sections (TDCS) - differential in energy and two solid angles (and so this is actually a fivefold differential cross section).

In this work we study electron-impact ionization of methane $\left(\mathrm{CH}_{4}\right)$ at intermediate incident energies. $\mathrm{CH}_{4}$ is the most prevalent greenhouse gas emitted on earth from human and animal activities, and is associated with global warming and climate change. The methane molecule is the smallest hydrocarbon and has a carbon atom at the center that shares 4 bonds with the hydrogen atoms. In this paper, we examine ionization of the highest occupied molecular orbital (HOMO), which is the $1 t_{2}$ state (and which is a predominantly ptype orbital), as well as the next highest occupied molecular orbital (NHOMO), which is the $2 a_{1}$ state.

In recent years, theory has made impressive progress in describing the electronimpact ionization dynamics of atomic hydrogen, helium and other atoms with one or two electrons in their outer shells. These atoms can be modeled as quasi-one and quasi-two electron targets. The interactions are described using various close-coupling methods, including convergent close coupling $(\mathrm{CCC})^{1}$, exterior complex scaling $(\mathrm{ECS})^{2}$, time dependent close coupling (TDCC) ${ }^{3}$, and R-matrix methods ${ }^{4}$. The treatment of more complex targets such as the noble gases has been far more challenging in particular at low incident energies, but great advances have been recently achieved. For example, it was shown that both the B-spline R-matrix method with pseudostates, and the 3-body distorted wave (3DW) approximation give excellent agreement with full three-dimensional experimental data for ionization of neon at the relatively low incident-electron energy of $65 \mathrm{eV}^{5}$. The R-matrix method also yields relatively good agreement with experimental data for ionization of argon using similar kinematical conditions to the neon experiments, and it is found that this agreement is better than for the $3 \mathrm{DW}$ model $^{6}$.

The multi-center nature of molecular targets makes the theoretical treatment of ionization processes significantly more challenging. At the present time the close coupling 
approaches have only been applied to ionization of $\mathrm{H}_{2}$ but not to larger molecules. In recent work, several atomic methods have been adapted to study molecular targets. Ionization of methane, in particular, has been investigated within variants of a three-Coulomb wave model $^{7-9}$, distorted wave approaches ${ }^{10-14}$ and the complex Kohn variational method ${ }^{15}$. Two other approaches, used in the present paper, are the following. The 3DW approach for atoms has been generalized to molecules and this M3DW (molecular three-body distorted wave) approximation has been successful for several different targets over a large range of energies ${ }^{16,17}$. The GSF approach developed initially for atoms ${ }^{18}$ has been adapted to deal with small polyatomic molecules ${ }^{19}$, and has been applied to study single ionization by both photon $^{20}$ and electron ${ }^{21}$ impact.

There have been a few previous $(\mathrm{e}, 2 \mathrm{e})$ studies of electron-impact ionization of methane for lower incident electron energies. Nixon et al. ${ }^{22}$ examined equal-energy final state electrons with energies between $2.5 \mathrm{eV}$ and $25 \mathrm{eV}$ in a coplanar asymmetric geometry. Normally, TDCS measurements exhibit a large peak near the momentum transfer direction (called the binary peak) and a small peak in the opposite direction (called the recoil peak). Nixon et $a l .{ }^{22}$ found that the recoil peak became larger than the binary peak with decreasing energy. Following from this work, Nixon et al. ${ }^{23}$ compared the TDCS for ionization of methane and neon in the perpendicular plane, for final state electrons of equal energy ranging between $2.5 \mathrm{eV}$ and $30 \mathrm{eV}$. For neon, the experimental measurement exhibited a double peak at high energy and a single peak at lower energy, while for methane the shape of the TDCS was more complicated. $\mathrm{Xu}$ et al. ${ }^{10}$ reported experimental and theoretical results for $54 \mathrm{eV}$ electron-impact single ionization of the $\mathrm{CH}_{4} \mathrm{HOMO}$ state in both coplanar and perpendicular planes. Although they found qualitative agreement between theory and experiment, the agreement was not as good. The M3DW results presented in that work used the orientation averaged molecular orbital (OAMO) approximation. Without the latter approximation, the M3DW calculations reported later by Chaluvadi et al. ${ }^{11}$ were in much better agreement with experiment.

Measurements in coplanar asymmetric geometries for the larger incident electron energy of $500 \mathrm{eV}$ have been performed by the Orsay group ${ }^{7}$. Except possibly in the recoil region, the TDCS for the ionization from both the $1 t_{2}$ and $2 a_{1}$ states have been well reproduced by a number of theoretical treatments ${ }^{7,9,12,13,15,19,21}$.

For an incident energy of $250 \mathrm{eV}$, Isik et al. ${ }^{24}$ measured the TDCS for the ionization of the $1 t_{2}$ state, with an ejected energy of $50 \mathrm{eV}$ again in coplanar asymmetric geometries. Published calculations could reproduce the typical binary plus recoil shapes, but not the 
binary to recoil peak ratios ${ }^{8,9,21}$. By varying the kinematical and geometrical configurations, a clear double peak structure in the binary region was predicted theoretically within the GSF model $^{21,25}$. Such a double structure, attributed to the p-type character of the ionized molecular orbital, has been observed also in experiments on $\mathrm{N}_{2} \mathrm{O}^{26}, \mathrm{H}_{2} \mathrm{O}^{27}$ and $\mathrm{CO}_{2}{ }^{28}$.

It is the purpose of this paper to explore the angular distributions of the TDCS corresponding to the ionization of the HOMO and $\mathrm{NHOMO}$ states of $\mathrm{CH}_{4}$, with a focus on the single or double peak structure in the binary region. To this end, we consider an incident energy of $250 \mathrm{eV}$ and coplanar asymmetric geometries. We report several cross section measurements from two different laboratories and compare their results with calculations performed within the M3DW and GSF models.

A schematic of the coplanar geometry is shown in figure 1. The faster final state electron with energy $E_{a}$ and momentum $\mathbf{k}_{a}$ is detected at a fixed angle $\theta_{a}$ in the scattering plane (we call this electron the 'scattered' electron for convenience). The slower final state electron with energy $E_{b}$ and momentum $\mathbf{k}_{b}$ is detected at an angle $\theta_{b}$ that varies around the plane in a clockwise direction (we call this electron the 'ejected' electron).

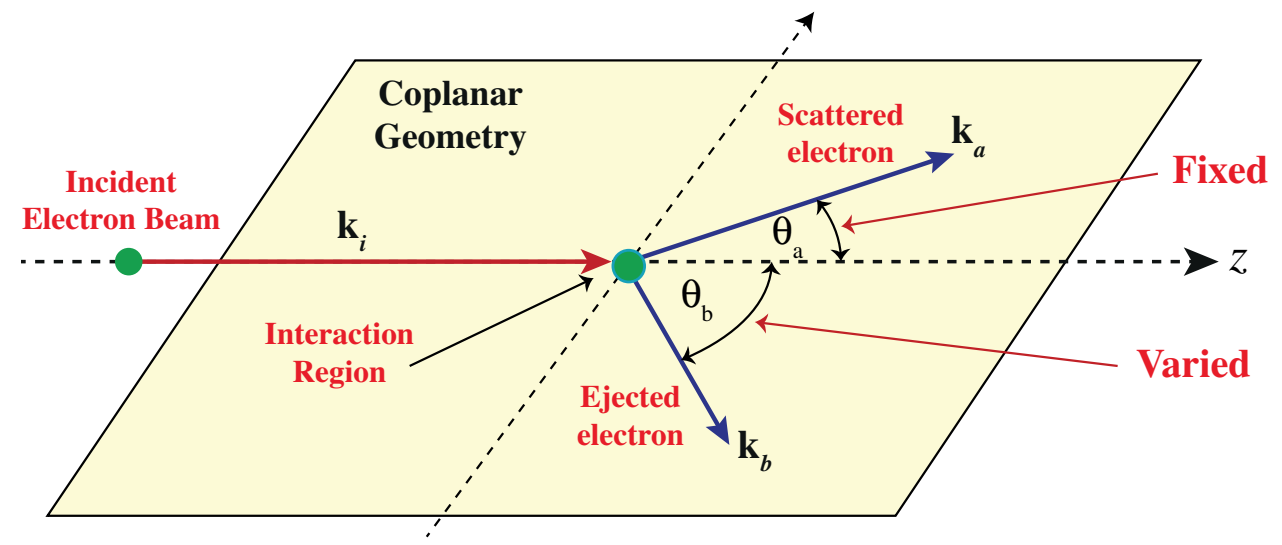

Figure 1. (color online) Schematic of the coplanar geometry. The incident electron has energy $E_{i}$ and momentum $\mathbf{k}_{i}$. The scattered electron has energy $E_{a}$, momentum $\mathbf{k}_{a}$, and is detected at a fixed angle $\theta_{a}$. The ejected electron has energy $E_{b}$, momentum $\mathbf{k}_{b}$, and its detection angle $\theta_{b}$ is varied.

The momentum transfer direction is determined by the momentum of the incident electron and the scattered electron, and is given by:

$$
\mathbf{q}=\mathbf{k}_{i}-\mathbf{k}_{a}
$$




\section{THEORY}

\section{A. M3DW theory}

The M3DW model has been presented in Ref. [17] so we provide only a brief outline of the theory here. The direct scattering amplitude is given by

$$
T_{d i r}=\left\langle\chi_{a}^{-}\left(\mathbf{k}_{a}, \mathbf{r}_{a}\right) \chi_{b}^{-}\left(\mathbf{k}_{b}, \mathbf{r}_{b}\right) \mathrm{C}_{a b}\left(\mathbf{k}_{a b}, \mathbf{r}_{a b}\right)\left|\mathrm{V}_{i}-U_{i}\right| \phi_{D y}\left(\mathbf{r}_{b}, \mathbf{R}\right) \chi_{i}^{+}\left(\mathbf{k}_{i}, \mathbf{r}_{a}\right)\right\rangle
$$

where $\chi_{i}^{+}\left(\mathbf{k}_{i}, \mathbf{r}_{a}\right)$ is a continuum-state distorted wave and the $(+)$ indicates outgoing wave boundary conditions. $\chi_{a}^{-}\left(\mathbf{k}_{a}, \mathbf{r}_{a}\right)$ and $\chi_{b}^{-}\left(\mathbf{k}_{b}, \mathbf{r}_{b}\right)$ are the scattered and ejected electron distorted waves with incoming wave boundary conditions. The factor $\mathrm{C}_{a b}\left(\mathbf{k}_{a b}, \mathbf{r}_{a b}\right)$ is the final state Coulomb-distortion factor between the two electrons, normally called the post collision interaction (PCI). $\mathbf{r}_{a b}$ is the relative separation between the two electrons and $\mathbf{k}_{a b}$ is the relative momentum. The term $V_{i}$ is the initial state interaction between the projectile electron and the target, and $U_{i}$ is a spherically symmetric approximation for $V_{i}$. Finally $\phi_{D y}\left(\mathbf{r}_{b}, \mathbf{R}\right)$ is the initial bound-state wave function which depends both on position vector $\mathbf{r}_{b}$ and the orientation of the molecule designated by $\mathbf{R}$. It is taken here as the Dyson molecular orbital for the active electron, which is calculated using density function theory with a $\mathrm{B} 3 \mathrm{LYP} / \mathrm{TZ2P}$ basis set. Brion et al. ${ }^{29}$ have recently shown that this type of wavefunction is accurate for $\mathrm{CH}_{4}$. For a given orientation $\mathbf{R}$ with respect to the laboratory frame, the TDCS is then obtained from

$$
\sigma^{T D C S}(\mathbf{R})=\frac{1}{(2 \pi)^{5}} \frac{k_{a} k_{b}}{k_{i}}\left(\left|T_{d i r}(\mathbf{R})\right|^{2}+\left|T_{e x c}(\mathbf{R})\right|^{2}+\left|T_{d i r}(\mathbf{R})-T_{e x c}(\mathbf{R})\right|^{2}\right)
$$

where the exchange amplitude $T_{e x c}$ is of the same form as Eq. (2), except that the $\mathbf{r}_{a}$ and $\mathbf{r}_{b}$ coordinates in the final state wave function are exchanged.

\section{B. GSF Theory}

The adaptation of the GSF approach ${ }^{18}$ to single ionization of molecules has been developed in Ref. [19] and has been applied to small molecules in photon ${ }^{20}$ and electron ${ }^{21}$ impact studies. The spectral approach is based on using a basis set of Generalized Sturmian Functions (GSFs) that are, by definition, solutions of a two-particles Sturm-Liouville problem. One important characteristic of GSFs is that they can be tailored to contain suitable physical properties, making them efficient for a given scattering problem. All basis set 
functions can be chosen to have, in particular, a common preselected asymptotic behaviour that is appropriate to describe a given scattering state. In single ionization of a neutral target, for example, an ejected electron will have a specific energy and feel a unit charge at large distances. GSF's have been used successfully to study double ionization of helium induced by photons $^{30}$, electrons ${ }^{31,32}$ or proton ${ }^{33}$ impact.

The extension to study electron-impact ionization of small molecules was made making some approximations, such as the frozen core and the single active electron approximation. In the present calculations we use a one-centre expansion for the description of the initial state of methane and take the wave functions tabulated by Moccia ${ }^{34}$. Using a set of Euler angles, this initial state is rotated in space in order to incorporate the random target orientation, which is not resolved experimentally. Within a first order perturbative approximation, the incident and scattered electrons are described by plane waves, and the molecular potential is described through a static exchange approximation. For a given molecular orientation $\mathbf{R}$, the ionization process is studied solving a first order driven equation (as described in Refs. [19,21]) whose solution corresponds to the ejected electron wave function. To obtain this molecular single continuum, we propose an expansion in a set of GSFs with a Coulomb asymptotic behaviour corresponding to exactly the $\mathrm{E}_{\mathrm{b}}$ energy. Because of this property, the ionization amplitude (and thereafter the TDCS as in Eq. (3)) is extracted directly from the expansion coefficients; there is no need to evaluate a transition matrix element (six-dimensional integral) as for example through Eq. (2) in the M3DW method.

For the treatment of coplanar asymmetric geometries in which we have a relatively fast incident electron and an ejected electron that carries a relatively small energy $\left(E_{b}<<E_{a}\right)$, we neglect exchange effects. The calculated TDCSs within our first-Born GSF approach possess then an intrinsic symmetry with respect to the momentum transfer direction.

\section{Orientation averaging}

Whether in the M3DW or the GSF approach, the TDCS are calculated for a given molecular orientation $\mathbf{R}$. Since the target molecules are randomly oriented in the experiments, we need to take an average of the theoretical TDCS over all possible orientations $^{11,21}$, so that:

$$
\sigma=\frac{\int \sigma^{\mathrm{TDCS}}(\mathbf{R}) \mathrm{d} \Omega_{R}}{\int d \Omega_{R}} .
$$




\section{EXPERIMENT AND RESULTS}

\section{A. The Afyon experiment and results}

The measurements in Afyon have been carried out using an electron-electron coincidence spectrometer. A detailed description of the apparatus and its applications to ionization of atomic and molecular targets is given in references [24], [35] and [28] and so only a brief description is presented here. The spectrometer comprises an electron gun, two rotatable electrostatic hemispherical electron analyzers equipped with channel electron multipliers (CEM's), and a Faraday cup. The spectrometer is located in a cylindrical stainless steel vacuum chamber. The pressure in the chamber was maintained around $2 \times 10^{-6}$ torr during data accumulation. The spectrometer operated with an electron current of $\sim 1 \mu \mathrm{A}$ and had an energy resolution of $\sim 0.6 \mathrm{eV}$. The gas jet and electron gun are fixed to produce orthogonal beams whose intersection defines the collision center. The electron beam produced by the gun can be varied between $10 \mathrm{eV}$ and $450 \mathrm{eV}$, while producing a collimated beam of electrons having a diameter of $2 \mathrm{~mm}$ in the interaction region. Both ejected and scattered electrons were detected using hemispherical analyzers.

The Afyon data and theoretical TDCS results for $250 \mathrm{eV}$ electron-impact ionization of the $\mathrm{HOMO}$ state of $\mathrm{CH}_{4}$ are compared in figures 2 and 3 for ejected electron energies of 50 $\mathrm{eV}$ and $30 \mathrm{eV}$. The vertical arrows indicate the direction of momentum transfer $+\mathbf{q}$ and $-\mathbf{q}$. Since the absolute values of the experimental data were not determined, both theory and experiment have been normalized to unity at the binary peak in each plot. 


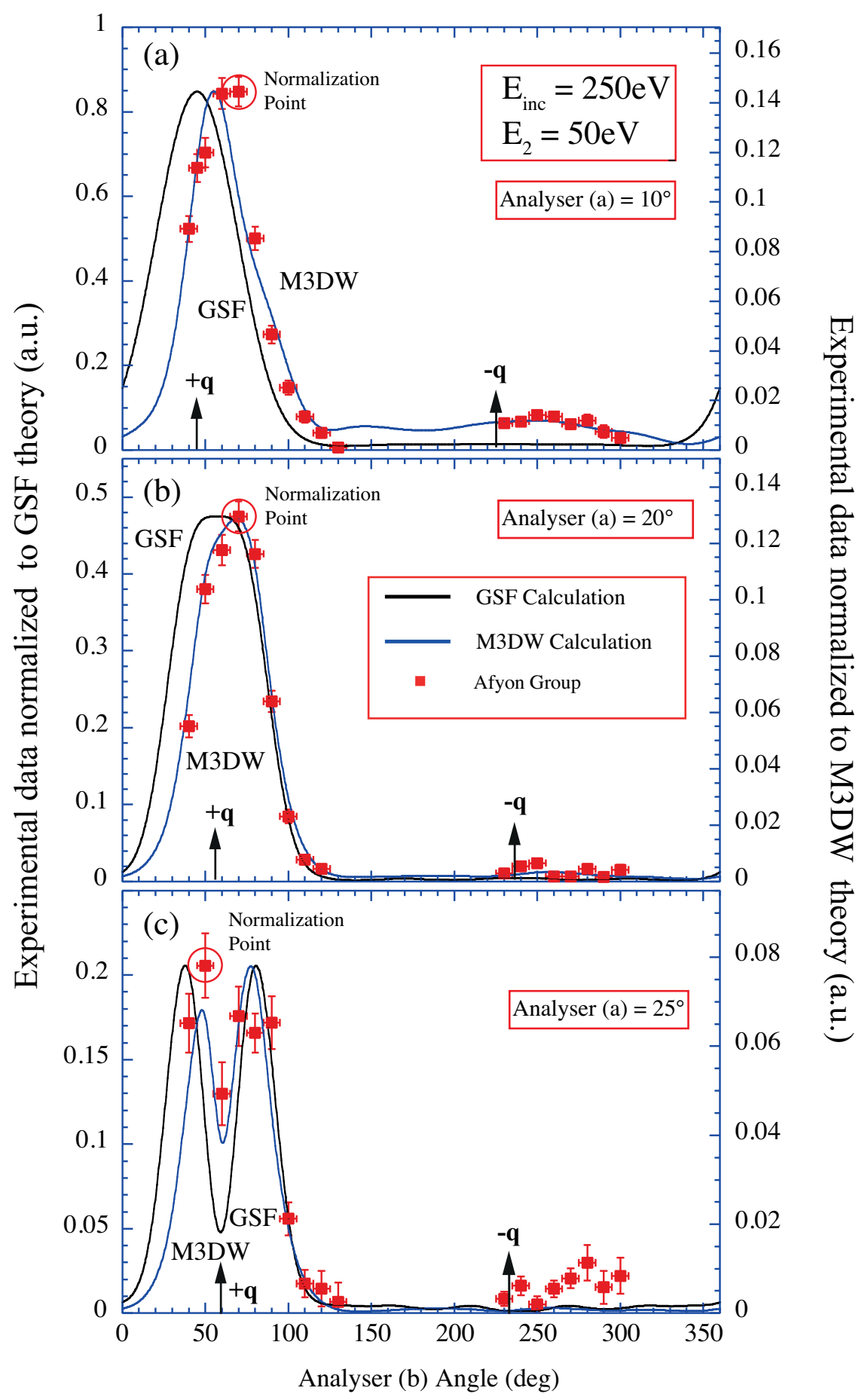

Figure 2. (color online) Comparison of experimental and theoretical TDCS for $250 \mathrm{eV}$ electronimpact ionization of the $1 t_{2}$ orbital of $\mathrm{CH}_{4}$. The ejected electron has an energy of $50 \mathrm{eV}$, and the scattered electron angles are as shown. (a) shows the results for analyser (a) at 10 ${ }^{\circ}$, (b) when set to $20^{\circ}$ and (c) for $25^{\circ}$. The data are measured in the Afyon laboratory and are normalized to the calculations in each panel, the left scale showing the GSF calculation (in atomic units) and the right scale the M3DW calculation (again in atomic units). The calculations differ in magnitude by a factor from 3 to 6, depending on the angle of analyser (a).

As seen from figure 2, where three different fixed electron scattering angles $\left(10^{\circ}\right.$, $20^{\circ 24}$, and $25^{\circ}$ ) are considered, both M3DW and GSF results are in reasonably good agreement with the shape of the experimental data (we can only compare shapes under these normalization conditions). By construction, the GSF calculation predicts a binary peak that is 
symmetric about the momentum transfer direction. For the two smaller scattering angles $\left(10^{\circ}, 20^{\circ}\right)$, both experiment and M3DW calculation have a peak at a larger angle than the momentum transfer direction, and this can be attributed to the final state electron-electron repulsion (i.e. PCI). The M3DW model contains PCI to all orders of approximation, and this produces very good agreement with experiment. There is a very small recoil peak found in the data for the smallest scattering angle $\left(\theta_{a}=10^{\circ}\right)$ that is also in good agreement with the M3DW results.

One of the interesting aspects of these results is the behavior of the binary peak with increasing projectile scattering angles. For the two smaller angles, there is a single peak near the momentum transfer direction $+\mathbf{q}$. For the largest scattering angle, there is a double peak with a fairly deep minimum at $+\mathbf{q}$. This type of behavior has previously been observed for ionization of the p-states of neon ${ }^{36}$, $\operatorname{argon}^{37}$, and xenon ${ }^{36}$. Whelan ${ }^{38}$ suggested that this minimum for $\mathrm{p}$-states can be explained by considering the plane wave impulse approximation (PWIA). In this approximation, the TDCS is proportional to the square of the momentum space wavefunction. For the $+\mathbf{q}$ direction, the momentum space wavefunction for $\mathbf{p}$-states is zero, and so in this approximation the TDCS must also be zero. The exact zero in the PWIA becomes a minimum for more accurate calculations. This interpretation can be considered under high incident energy and close to bound Bethe ridge conditions. When moving away from such regimes, in particular in the coplanar asymmetric configurations investigated in the present study, the situation is more difficult to interpret. Indeed, the ejected and scattered electrons play different roles since they have very different momenta; the two theoretical models are thus more complex and the cross section does not reduce to the square of the pelectron momentum distribution. To our knowledge no one has suggested a physical or kinematical explanation why the double peak is not always observed in the experimental data in such asymmetric energy regimes.

Haynes and Lohmann ${ }^{37}$ measured p-state ionization of argon for $113.5 \mathrm{eV}$ incident electrons and for a fixed scattering angle of $15^{\circ}$. They found a single peak for ejected electrons with $10 \mathrm{eV}$, and a double peak at lower energies. Stevenson et al. ${ }^{39}$ performed a similar experiment for ionization of argon at $200 \mathrm{eV}$ also with a fixed scattering angle of $15^{\circ}$. These experiments found a single peak for $20 \mathrm{eV}$ ejected electrons and a double peak for 10 $\mathrm{eV}$ and lower. Ren et $a .^{6}{ }^{6}$ reported measurements for ionization of the p-state of argon at 66 $\mathrm{eV}$ with scattering angles of $10^{\circ}, 15^{\circ}$, and $20^{\circ}$ and ejected electron energies of 3,5 and 10 $\mathrm{eV}$. For 5 and $10 \mathrm{eV}$ electrons, they found a single peak for all three scattering angles. However, for ejected electrons at $3 \mathrm{eV}$ they found a single peak for $10^{\circ}$ scattering and double 
peaks for $15^{\circ}$ and $20^{\circ}$. From these observations in argon, the trend seems to suggest double peaks form with decreasing ejected electron energy and fixed scattering angle. In one case, a double peak occurred with fixed ejected electron energy and increasing scattering angle, and in two cases no double peak was found with increasing scattering angle. However for neon, which is isoelectronic to $\mathrm{CH}_{4}$, Stevenson et al. ${ }^{36}$ found double peaks for $150 \mathrm{eV}$ incident electrons, $10 \mathrm{eV}$ ejected electrons and projectile scattering angles of $5^{\circ}, 10^{\circ}$, and $15^{\circ}$. This differs from the argon measurements, and so there does not appear to be a general trend throughout the targets that have been studied so far.

A similar behavior has been observed for ionization of $\mathrm{CO}_{2}$ at $250 \mathrm{eV}$ with $37 \mathrm{eV}$ ejected electrons and scattered electron angles of $10^{\circ}, 20^{\circ}$, and $30^{\circ}$. For this case, experiment found a single peak at $10^{\circ}$, a double peak at $20^{\circ}$, and a single peak again at $30^{\circ}$. The M3DW model predicted a single peak at $10^{\circ}$ and double peak at $20^{\circ}$ in agreement with experiment, however the model also predicted a double peak at $30^{\circ}$ contrary to the data. For previous $\mathrm{CH}_{4}$ measurements, Lahmam-Bennani et al. ${ }^{7}$ measured ionization of the $1 t_{2}$ state for $500 \mathrm{eV}$ incident electrons for a fixed projectile scattering angle $\left(6^{\circ}\right)$ but for three different ejected energies. They observed a hint of a double binary peak for $12 \mathrm{eV}$ ejected electrons, but a clear single peak for 37 or $74 \mathrm{eV}$. Xu et al. ${ }^{10}$ considered $54 \mathrm{eV}$ incident electrons, three pairs of ejected electron energies, and five projectile scattering angles ranging from $15^{\circ}$ to $55^{\circ}$ (i.e. 15 different data sets). Although no evidence for a double binary peak was seen in the data for angles larger than $20^{\circ}$, there was some indication of a double peak for $15^{\circ}$ and $20^{\circ}$. However, in previous atomic measurements, the minimum was either at or very close to the momentum transfer direction such as found here. By contrast, the minimum in the data from reference [10] occurred at much larger angles, so this double peak structure probably results from a different physical effect. As a result, although it is well known that the $1 t_{2}$ state of methane has a p-type momentum distribution ${ }^{29}$, previous experiments have not clearly observed a double peak structure in the TDCS angular distribution.

Figure 3 compares experimental data from the Afyon group with theory for $30 \mathrm{eV}$ ejected electrons and two fixed angles of $10^{\circ}$ and $20^{\circ}$. Again both calculations are in reasonably good agreement with the normalized data. For $\theta_{a}=10^{\circ}$, the experimental binary peak is shifted to larger angles relative to the momentum transfer direction, in agreement with the M3DW calculation. However, agreement between the M3DW theory and experiment is not as satisfactory as it was for the higher ejected energy. Again the single binary peak develops into a double peak with increasing scattering angle $\theta_{a}$. However for $\theta_{a}=20^{\circ}$, experiment shows that the larger angle peak is smaller in amplitude. This is in contrast with 
the theoretical results: the GSF predicts equal magnitudes for the twin peaks (as a direct consequence of the first Born model), whereas the M3DW calculation predicts a larger peak at the higher angle. For both ejected electron energies, the minimum is very close to the momentum transfer direction as predicted.

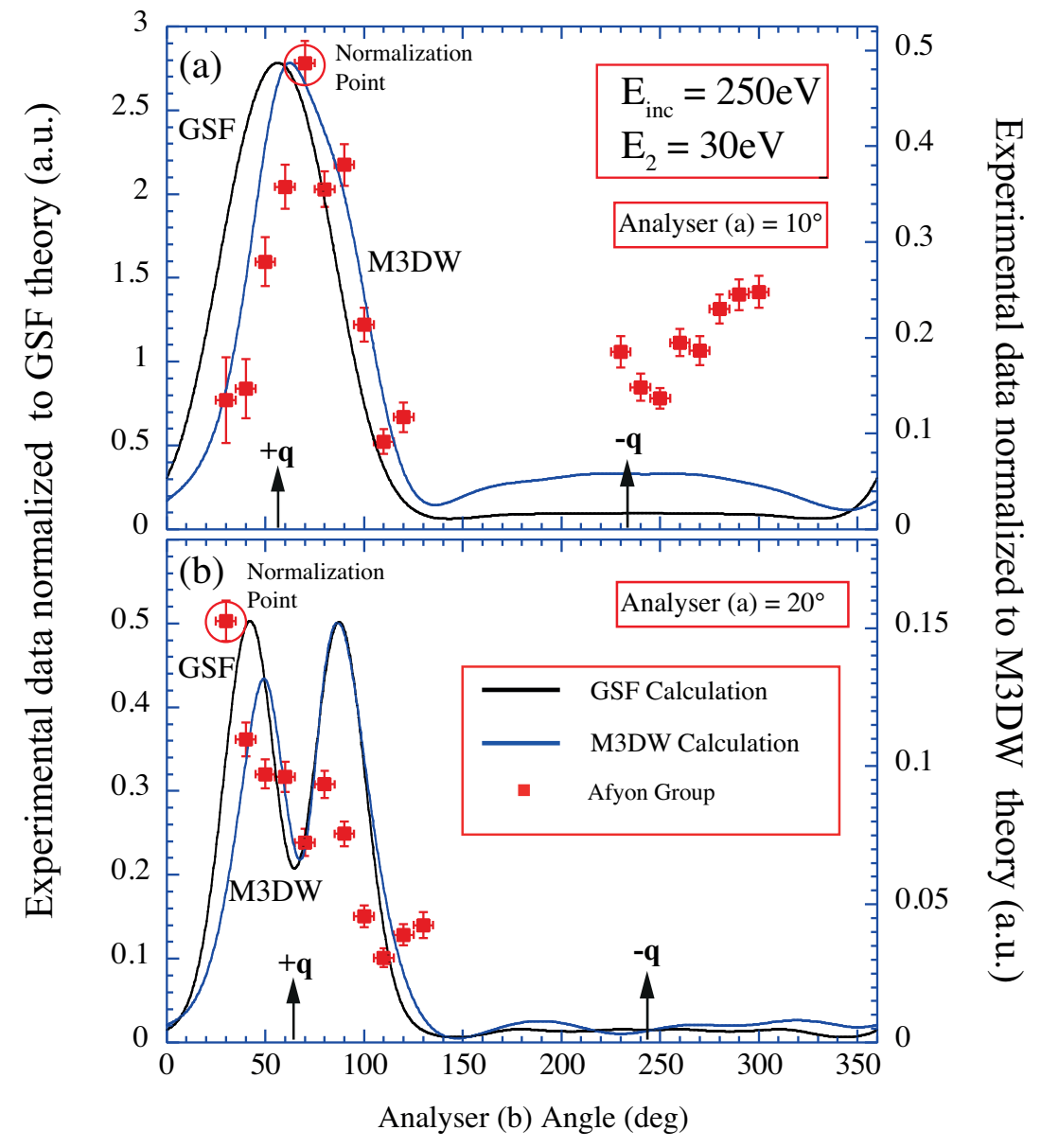

Figure 3. Same as figure 2, now for an ejected electron energy of $30 \mathrm{eV}$. (a) shows the results for analyser (a) at $10^{\circ}$, (b) shows the results for $20^{\circ}$.

We have also performed a detailed theoretical investigation of the behavior of the binary peak as a function of the fast electron scattering angle for several fixed ejected electron energies, including 50 and $30 \mathrm{eV}$. As an example, figure 4 presents a surface plot of $\mathrm{M} 3 \mathrm{DW}$ results for the $50 \mathrm{eV}$ case as a function of $\theta_{a}$ as this angle is varied from $10^{\circ}$ to $36^{\circ}$, with $\theta_{b}$ being the ejected electron angle. The single binary peak near $+\mathbf{q}$ for small $\theta_{a}$ is seen to develop into a double peak, with a deep minimum in the momentum transfer direction for $\theta_{a} \approx 22^{\circ}$ due to the valley formed with increasing $\theta_{a}$. For larger scattering angles, this valley develops back into a peak, so that the TDCS again is again a single peak at $\theta_{a} \approx 34^{\circ}$.

To obtain a better understanding of this double binary peak structure (and to get additional experimental verification), the experimental group in Manchester, UK performed a 
more detailed examination of the binary peak as a function of the faster electron scattering angle, and these results are presented in the next section.

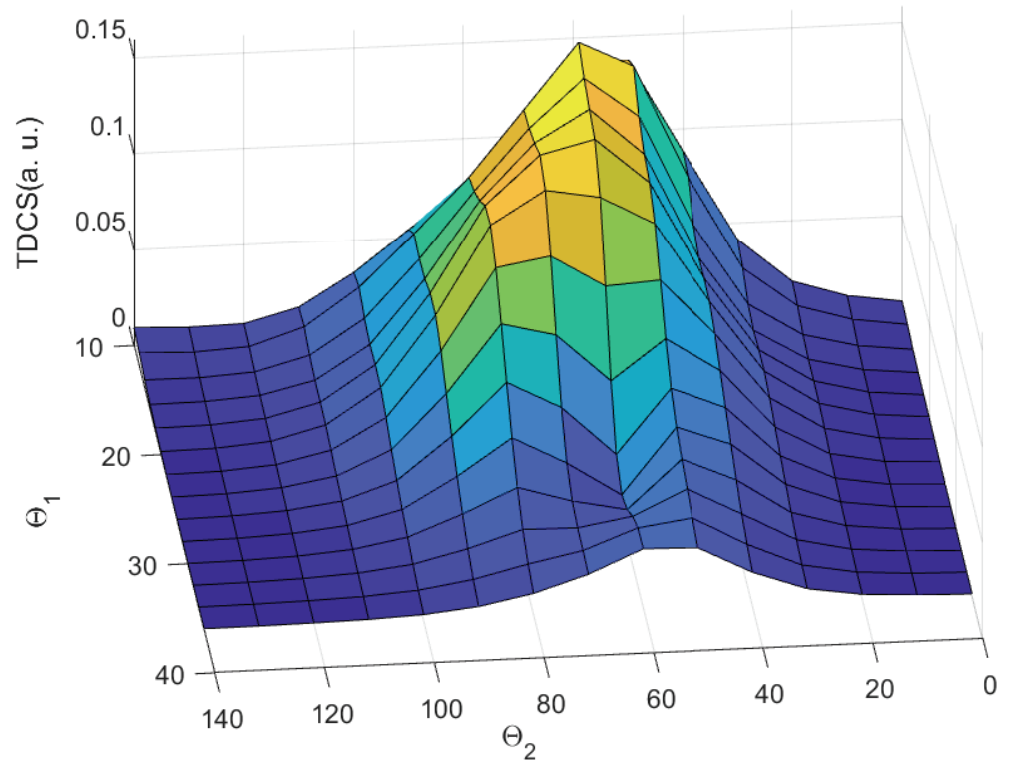

Figure 4. (color online) Surface plot of the M3DW TDCS for $\theta_{a}$ ranging between $10^{\circ}$ and $36^{\circ}$. The ejected electron angle in the scattering plane is $\theta_{b}$.

\section{B. The Manchester experiment and results}

The apparatus used to produce the experimental data from Manchester has been detailed previously ${ }^{40-42}$, so only a brief description is given here. This (e,2e) spectrometer comprises an electron gun, two electron energy analyzers, a Faraday cup and an atomic/molecular beam source, and so is similar to the Afyon apparatus. However in the Manchester spectrometer electron-impact single ionization coincidence events can be measured either in a coplanar geometry as adopted here, or in a non-coplanar geometry where the electron gun is rotated out of the detection plane spanned by the analyzers. The electron gun comprises a two-stage electrostatic lens that delivers a collimated electron beam to the interaction region with beam energy between $20 \mathrm{eV}$ and $300 \mathrm{eV}$, and with a beam current up to $5 \mu \mathrm{A}$. Scattered and ejected electrons are detected in coincidence by two electron analyzers. These use triple cylindrical lenses to image electrons emerging from the interaction region onto the entrance apertures of hemispherical energy selectors, the energy selected electrons again being detected by channel electron multipliers.

A Faraday cup is positioned on the opposite side of the interaction region to the electron gun. This cup measured the electron beam current and also reduced scattered electrons from the outgoing electron beam. In the coplanar geometry used here (figure 1), the physical size of the gun, analyzers and cup normally restricts the angular detection range 
from $\theta_{a, b}=35^{\circ}$ to $135^{\circ}$ for this system. However in the present work, the higher electron gun energy of $250 \mathrm{eV}$ allowed a smaller Faraday cup to be installed, so that forward angles of $\theta_{a, \min }=20^{\circ}$ and $\theta_{b, \min }=27.5^{\circ}$ could be reached.

A high-purity beam of $\mathrm{CH}_{4}$ was delivered by a platinum-iridium gas needle into the interaction region. The spectrometer was evacuated to a base pressure of $\sim 10^{-7}$ torr using a turbo-molecular pump. The chamber pressure was set to $1 \times 10^{-5}$ torr when the experiment was operating. An electron beam current of $1 \mu \mathrm{A}$ was chosen, which allowed a good coincidence signal to noise ratio to be obtained with the coincidence peak easily resolvable above background random counts. The Manchester spectrometer is fully controlled and optimized using new computer-controlled power supplies and new Labview code ${ }^{43}$, and so the analyzers were automatically tuned each time they moved to a new angle. The electrostatic voltages, beam current and gas pressure were routinely logged, and were adjusted to minimize changes in the operating conditions whilst maximizing coincidence yield.

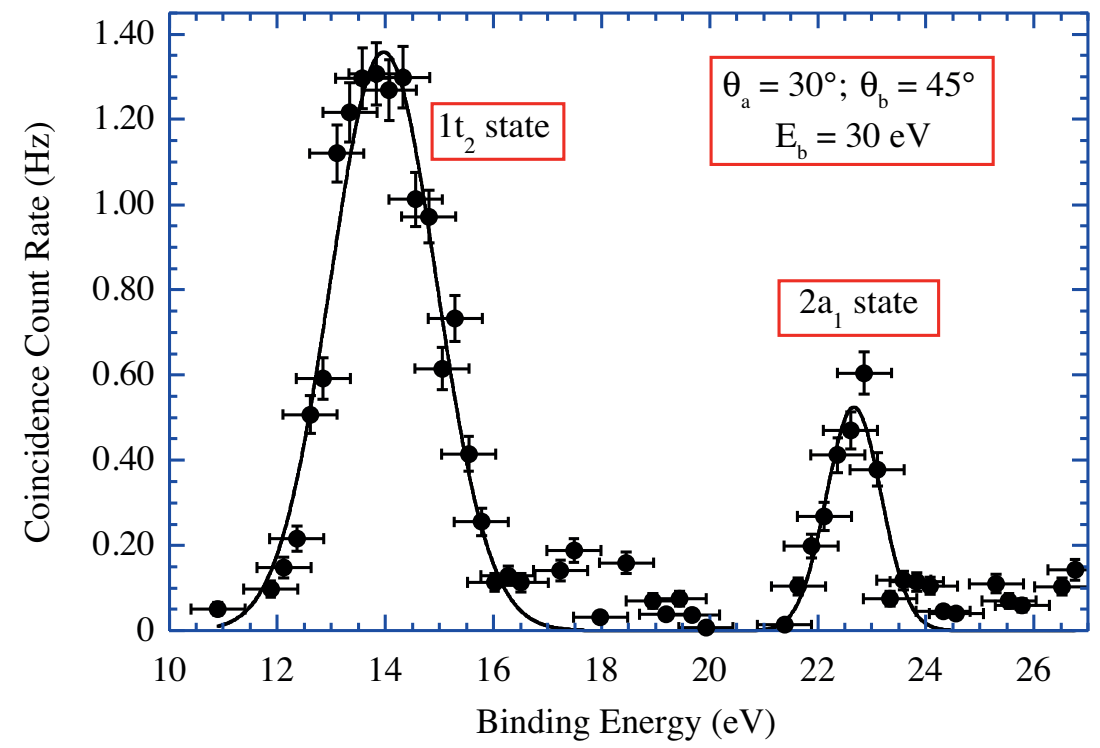

Figure 5. (color online) Binding energy spectrum obtained in the Manchester laboratory showing both $1 t_{2}$ and $2 a_{1}$ orbitals for $\theta_{a}=30^{\circ} \& \theta_{b}=45^{\circ}$. The orbitals are well-resolved and are fitted to Gaussians to establish their peak energy and width.

The data presented here studied ionization from both $1 t_{2}$ and $2 a_{1}$ orbitals. To ensure the experiment was correctly operating, a binding energy scan was performed at regular intervals by holding the analyzer energies fixed and scanning the incident electron energy. Figure 5 shows a typical spectrum from one of these scans. The two orbitals are clearly resolved, and are found to be separated by $\sim 9 \mathrm{eV}$. For the current experiments, the analyzers were operated in an asymmetric coplanar geometry, with analyzer (a) held at a fixed angle to 
detect the high-energy "scattered" electrons. Analyzer (b) was set to detect the low energy "ejected" electrons, and swept around the plane through $\theta_{b}=27.5^{\circ}$ to $130^{\circ}$ in $2.5^{\circ}$ steps. Coincidence data were accumulated for 1000 seconds at each angle, and up to 252 measurements were taken for each set at each fixed value $\theta_{a}$. The accumulated data at each angle $\theta_{b}$ were then averaged, and the statistical error used to assign an uncertainty. The fixed angles ranged from $\theta_{a}=20^{\circ}$ to $30^{\circ}$ in $2.5^{\circ}$ steps. Two experiments were carried out for the $1 t_{2}$ orbital using outgoing electron energies of $E_{b}=30 \mathrm{eV}$ and $50 \mathrm{eV}$, while maintaining the incident energy at $250 \mathrm{eV}$. For comparison, an additional set of data was acquired for the $2 a_{1}$ orbital with $E_{b}=30 \mathrm{eV}$ at a fixed angle $\theta_{a}=25^{\circ}$.

Like in the Afyon experiment, the Manchester data is not measured on an absolute scale, and so was normalized to a single point from both theories (see figures 6 and 7). The data were however inter-normalized for different fixed angles $\theta_{a}$ by taking 20 individual relative measurements at set angles, so that 100 inter-normalization measurements were taken for each energy $E_{b}$. The peak at $\theta_{a}=20^{\circ}$ was normalized to the calculations, since this was the strongest signal in each experiment. For the $2 a_{1}$ case, the measured peak of the data was normalized to theory as shown in figure 8 . In both sets of measurements, the vertical error bars represent the standard error derived from averaging multiple measurements at each angle, and the horizontal error bars represent the uncertainty due to the angular resolution of the spectrometer.

Figures 6 and 7 show both Manchester and Afyon data sets for the cases where the geometrical configurations overlapped. The two experimental measurements are in excellent agreement for $50 \mathrm{eV}$ ejected electrons at the two overlapping scattered electron angles of $20^{\circ}$ and $25^{\circ}$. For the case of $30 \mathrm{eV}$ ejected electrons and $\theta_{a}=20^{\circ}$, there is also very good agreement between the two experiments for the larger angle peak, while there is some disagreement on the location of the smaller angle peak. Overall the agreement between the two (normalized) experiments is very good. 


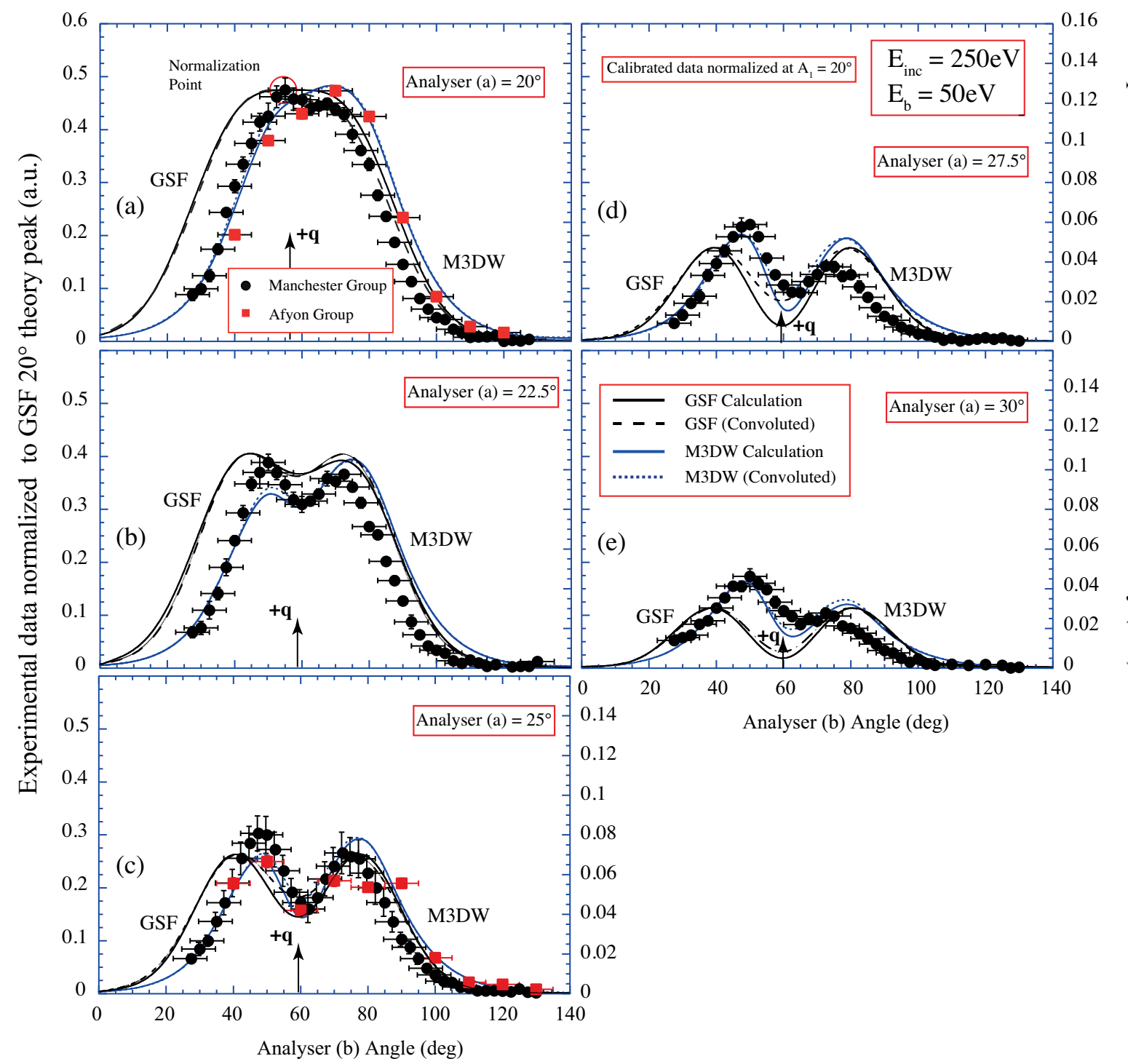

Figure 6. (color online) Experimental data from the Manchester and Afyon groups, compared to the $G S F$ and $M 3 D W$ theories for ionization from the $1 t_{2}$ orbital with an ejected electron energy of $50 \mathrm{eV}$. (a) is for analyser (a) at $20^{\circ}$, (b) for $22.5^{\circ}$, (c) for $25^{\circ}$, (d) for $27.5^{\circ}$ and (e) for $30^{\circ}$. The Manchester data are normalized to each theory as explained in the text, and the Afyon data are renormalized to the Manchester data, since inter-normalization was not carried out by this group. Note a factor of $\sim 4$ difference in magnitude is found between the TDCS obtained using the M3DW and GSF models, and so two vertical scales are used to represent the different calculations.

Comparing the two theories, it is seen that both consistently predict similar TDCS shapes for all measured cases. The M3DW is in good agreement with experiment for both the location of the peaks and the relative magnitude of the data at all angles $\theta_{a}$. It is again emphasized that the Manchester data is normalized to theory only at a single point when $\theta_{a}=20^{\circ}$, and so all measurements (a) to (e) as $\theta_{a}$ varies are directly linked to this single point of normalization. In a similar way to Figure 2, the M3DW calculation is in good agreement with all measurements for $50 \mathrm{eV}$ ejected electrons. This agreement is less satisfactory at $30 \mathrm{eV}$. When there are two peaks, both experiments predict that the larger angle peak has smaller intensity than the small angle peak. For the GSF calculation, the double peak intensities are the same by symmetry, and thus cannot reproduce this trend. By 
contrast the M3DW calculation has peak intensities that are different, however this model consistently overestimates the larger angle peak compared to the data.

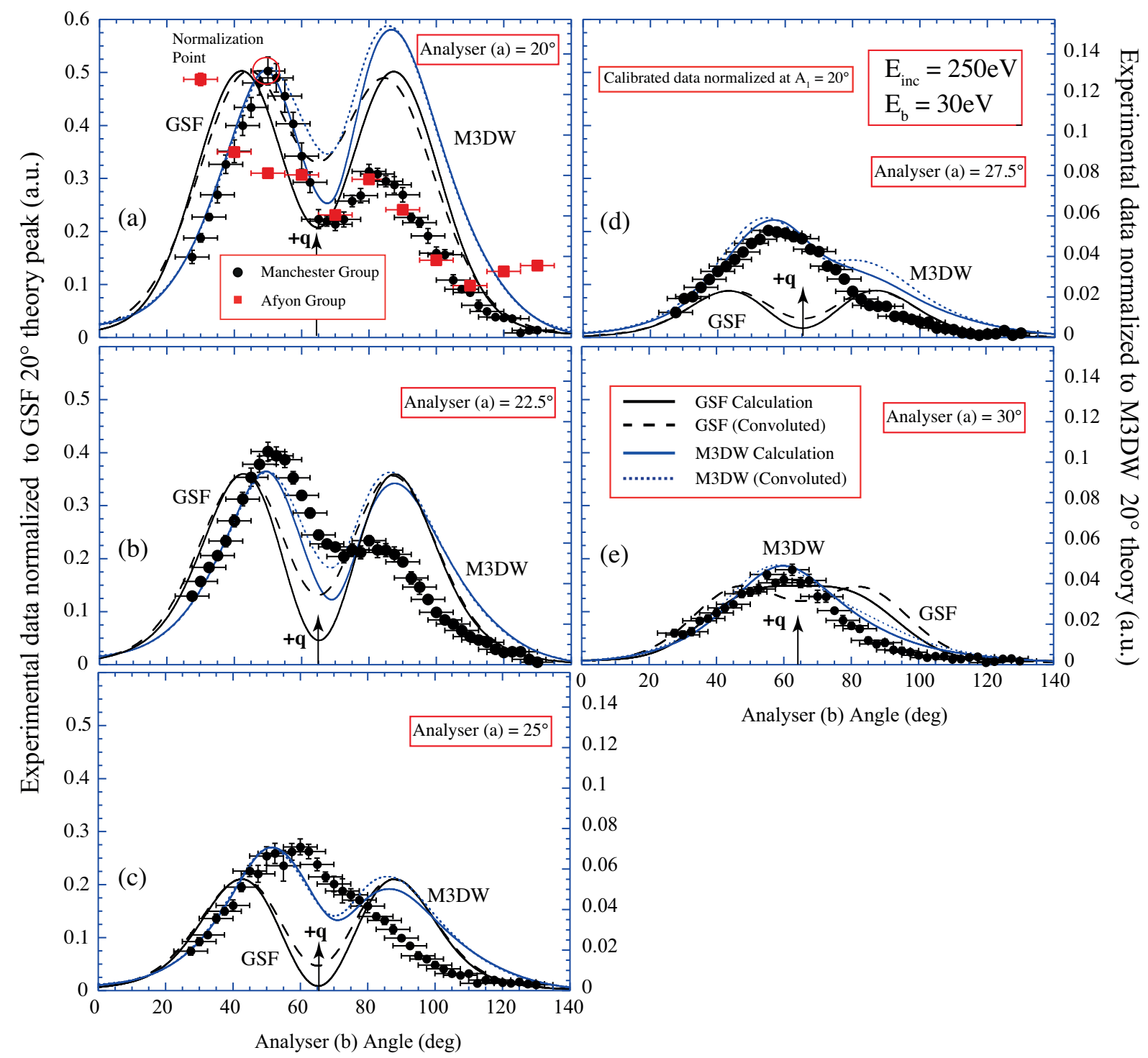

Figure 7. (color online) Experimental data from the Manchester and Afyon groups, compared to the $G S F$ and M3DW theories for ionization from the $1 t_{2}$ orbital with an ejected electron energy of $30 \mathrm{eV}$. (a) is for analyser (a) at 20 , (b) for $22.5^{\circ}$, (c) for $25^{\circ}$, (d) for $27.5^{\circ}$ and (e) for $30^{\circ}$. The Manchester data are normalized to each theory as explained in the text, and the Afyon data are renormalized to the Manchester data, since inter-normalization was not carried out by this group. Note a factor of $\sim 4$ difference in magnitude is found between the TDCS obtained using the M3DW and GSF models, and so two vertical scales are used to represent the different calculations.

The motivation for making detailed measurements in $2.5^{\circ}$ steps for $\theta_{a}$ was to monitor the evolution of the double peak structure with increasing scattering angles. For 50 $\mathrm{eV}$, it is seen that a single peak for $\theta_{a}=20^{\circ}$ evolved into two peaks with a fairly deep minimum between them by $\theta_{a}=25^{\circ}$. For increasing $\theta_{a}$, the larger angle second peak starts to reduce relative to the first and has almost gone by $\theta_{a}=30^{\circ}$. This is in relatively good agreement with the M3DW model. As a result, the experiment confirms the evolution of the TDCS shown in figure 4. 
By contrast, the $30 \mathrm{eV}$ case is quite different. The experiment shows that the higherangle peak in the double-peak structure is already negligible by $\theta_{a}=25^{\circ}$. For the M3DW theory, the second peak is essentially eliminated by $\theta_{a}=27.5^{\circ}$, and both theories predict it will completely disappear by $\theta_{a}=30^{\circ}$. It was initially thought that the lack of agreement with experiment for the $30 \mathrm{eV}$ case might be due to the theories being evaluated for a fixed $\theta_{a}$ while there is an angular uncertainty of $\pm 5^{\circ}$ in the experiment. To test this supposition, the theoretical results were convoluted using a Gaussian with a full width at half maximum that represents the angular acceptance angle of the apparatus. These convoluted M3DW and GSF results are shown as dotted and dashed curves respectively in figures 6 and 7. Clearly taking into account the angular resolution has little effect, and so cannot explain the observed differences between experiment and theory.
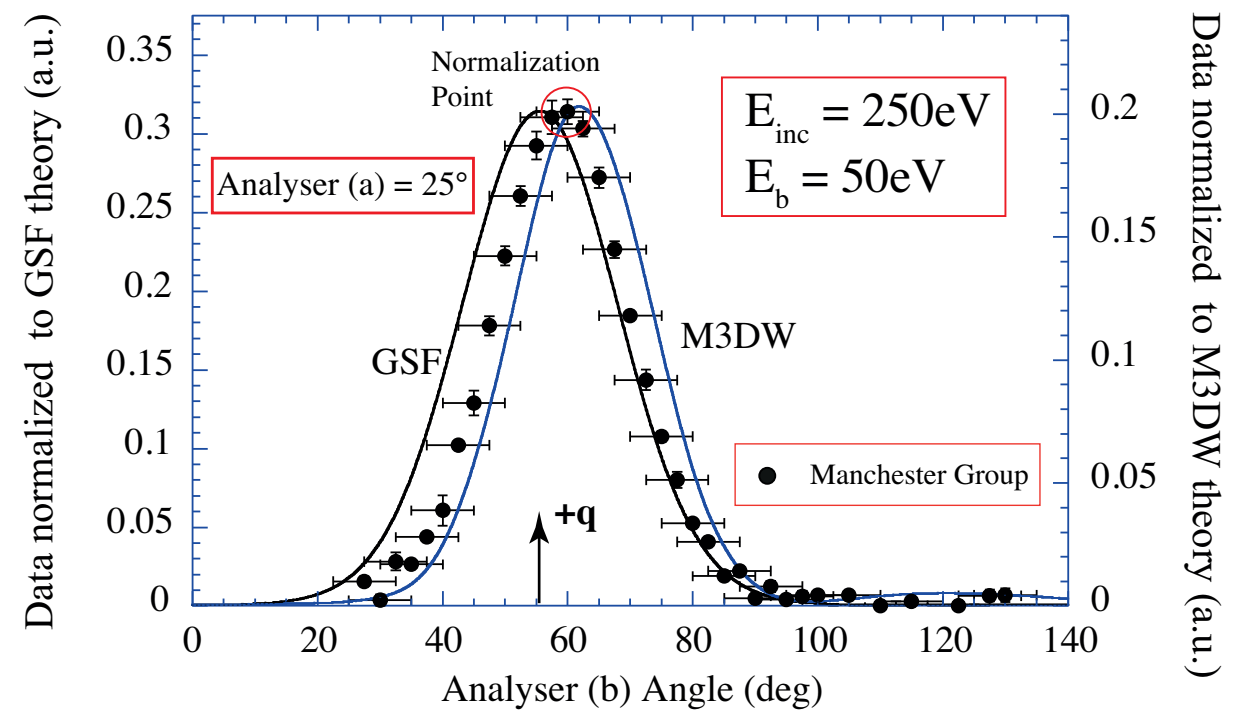

Figure 8. (color online) Comparison of the calculated TDCS in the GSF and M3DW models with data from Manchester for ionization from the $2 a_{1}$ orbital. The calculations have different magnitudes and so two vertical scales have been used to represent them.

Figure 8 compares theory and data from Manchester for ionization of the $2 a_{1}$ state, for an ejected electron energy of $50 \mathrm{eV}$ and with $\theta_{a}=25^{\circ}$. The $2 a_{1}$ state has an s-type momentum distribution ${ }^{29}$ and the TDCS reflects this, with no structure seen in either the experimental or theoretical angular distributions. Although the GSF predicts a peak at a smaller angle than seen in experiment and the M3DW predicts a peak at a larger angle, both theories essentially lie within the experimental uncertainty. No further measurements were made from this orbital, since it did not appear that there was much more that could be learned under these kinematic conditions. 


\section{CONCLUSIONS}

In this paper we have compared experimental measurements taken at two different laboratories with M3DW and GSF theoretical calculations, for electron-impact single ionization of the $1 t_{2}$ and $2 a_{1}$ states of molecular $\mathrm{CH}_{4}$. Overall, the measured cross sections were in very good agreement with each other. For the case of $50 \mathrm{eV}$ ejected electron energy, both calculations were in good agreement with the shape of the experimental data. For the 30 $\mathrm{eV}$ case, this agreement was not as satisfactory, but still reasonable. Compared to the GSF calculations, the M3DW results are in slightly better agreement with respect to the location of experimental peaks and to the relative magnitude of the Manchester data.

It is known that the $1 t_{2}$ state is predominantly p-type and we found, in the binary region, both single and double peaks that are characteristic of ionization from atomic $\mathrm{p}$-states. We examined closely the behavior of the binary peak structure as a function of the fast electron scattering angle for a fixed electron ejection energy. Experimentally, for electrons ejected with $50 \mathrm{eV}$ a single peak was seen for $\theta_{a}=20^{\circ}$ which evolved into a double peak with a fairly deep minimum around $\theta_{a}=25^{\circ}$. For larger $\theta_{a}$, the higher angle peak decreased in magnitude and had almost disappeared by $\theta_{a}=30^{\circ}$ (which was the largest angle measured). At this energy the experimental peak evolution was in good agreement with the theoretical predictions. For the case where the ejected electrons had $30 \mathrm{eV}$, a double peak at $\theta_{a}=20^{\circ}$ was seen to evolve into a single peak with increasing $\theta_{a}$. While these evolutions in the peaks are predicted by both theories, the experiments found that the decrease in amplitude of the larger angle peak happened more quickly with increasing $\theta_{a}$ than predicted by the theories. Although there have been several previous measurements for single ionization of $\mathrm{CH}_{4}$ by electron impact, this is the first clear observation of a double binary peak.

The Manchester group also measured the TDCS for ionization of the $2 a_{1}$ state for an ejected electron energy of $50 \mathrm{eV}$ and for $\theta_{a}=25^{\circ}$. A single symmetric binary peak with no additional structure was found, as is expected for ionization of an s-type orbital. Both theories predicted the same binary peak and both were within the experimental uncertainty. 


\section{ACKNOWLEDGEMENTS}

This work was partly supported by the US National Science Foundation under Grant. No. PHY-1505819, and by the National Natural Science Foundation of China under Grants No. 11174175. Computational work was performed with Institutional Computing resources made available through the Los Alamos National Laboratory. The Los Alamos National Laboratory is operated by Los Alamos National Security, LLC, for the National Nuclear Security Administration of the US Department of Energy under Contract No. DE-AC5206NA25396. The experimental work in Manchester was funded by EPSRC grant R120272.

\section{REFERENCES}

${ }^{1}$ I. Bray and A.T. Stelbovics, Phys. Rev. A 46, 9665 (1992).

${ }^{2}$ T. N. Rescigno, M. Baertschy, W. A. Isaacs, and C. W. McCurdy, Science 286, 2474 (1999).

${ }^{3}$ J. Colgan, M. S. Pindzola, and F. Robicheaux, J. Phys. B: At. Mol. Opt. Phys. 37, L377 (2004).

${ }^{4}$ O. Zatsarinny and K. Bartschat, Phys. Rev. A 85, 062710 (2012).

${ }^{5}$ X. Ren, S. Amami, O. Zatsarinny, T. Pfluger, M. Weyland, W. Y. Baek, H. Rabus, K. Bartschat, D.H. Madison, and A. Dorn, Phys. Rev. A 91, 032707 (2015).

${ }^{6}$ X. Ren, S. Amami, O. Zatsarinny, T. Pflüger, M. Weyland, A. Dorn, D. H. Madison, and K. Bartschat, Phys. Rev. A. 93, 062704 (2016).

${ }^{7}$ A. Lahmam-Bennani, A. Naja, E.M. Staicu Casagrande, N. Okumus, C. Dal Cappello, I. Charpentier and S. Houamer, J. Phys. B 42, 165201 (2009).

${ }^{8}$ M. Chinoune, S. Houamer, C. Dal Cappello and A. Galstyan, J. Phys. B 49, 205201 (2016).

${ }^{9}$ S. Houamer, M. Chinoune and C. Dal Cappello, Eur. J. Phys. D 71, 17 (2017).

${ }^{10}$ S. Xu, H. Chaluvadi, X. Ren, T. Pflüger, A. Senftleben, C. G. Ning, S. Yan, P. Zhang, J. Yang, X. Ma, J. Ullrich, D. H. Madison, and A. Dorn, J. Chem. Phys. 137, 024301 (11pp) (9 July 2012).

${ }^{11}$ H. Chaluvadi, C. G. Ning and D. H. Madison, Phys. Rev. A 89, 062712 ( 20 June 2014).

${ }^{12}$ I. Toth and L. Nagy, J. Phys. B 43, 135204 (2015). 
${ }^{13}$ M. Gong, X. Li, S. Bin Zhang, L. Liu, Y. Wu, J. Wang, Y. Qu and X. Chen, Phys. Rev. A 96, 042703 (2017).

${ }^{14}$ I. Toth, R.I. Campeanu and L. Nagy, Eur. Phys. J. D 69, 2 (2015).

${ }^{15}$ C.Y. Lin, C.W. McCurdy and T.N. Rescigno, Phys. Rev. A 89, 052718 (2014).

${ }^{16}$ O. Al-Hagan, C. Kaiser, D.H. Madison and A.J. Murray, Nature Physics 5, 59 (2009).

${ }^{17}$ D.H. Madison and O. Al-Hagan, J. Atomic, Molecular, and Optical Physics 2010, 367180, (2010).

${ }^{18}$ G. Gasaneo, L.U. Ancarani, D.M. Mitnik, J.M. Randazzo, A.L. Frapiccini and F.D. Colavecchia, Adv. Quantum Chem. 67, 153 (2013).

${ }^{19}$ C.M. Granados-Castro, Ph.D. thesis (Application of Generalized Sturmian Basis Functions to Molecular Systems), Université de Lorraine, Metz (2016).

${ }^{20}$ C.M. Granados-Castro, L.U. Ancarani, G. Gasaneo and D.M. Mitnik, Adv. Quantum Chem. 73, 3 (2016).

${ }^{21}$ C. M. Granados-Castro and L. U. Ancarani, Eur. J. Phys. D 71, 65 (2017).

${ }^{22}$ K. L. Nixon, A. J. Murray, H. Chaluvadi, C. Ning, and D. H. Madison, J. Chem. Phys. 134, 174304 (2011).

${ }^{23}$ K. L. Nixon, A.J. Murray, H. Chaluvadi, S. Amami, D. H. Madison and C. Ning, J. Chem. Phys. 136, 094302 (2012).

${ }^{24}$ N. Isik, M. Dogan and S. Bahceli, J. Phys. B 49, 065203 (2016).

${ }^{25}$ C. M. Granados-Castro and L. U. Ancarani, J. Phys.: Conf. Series 875, 062032 (2017).

${ }^{26}$ S.J. Cavanagh and B. Lohmann, J. Phys. B 32, L261 (1999).

${ }^{27}$ D.S. Milne-Brownlie, S.J. Cavanagh, B. Lohmann, C. Champion, P.A. Hervieux, J. Hanssen, Phys. Rev. A 69, 032701 (2004).

${ }^{28}$ Z. N. Ozer, E. Ali, M. Dogan, M. Yavuz, O. Alwan, A. Naja, O. Chuluunbaatar, B. B. Joulakian, C. Ning, J. Colgan, and D.H. Madison, Phys. Rev. A 93, 062707 (2016.).

${ }^{29}$ C.E. Brion, Saul Wolfe, Zheng Shi, Glyn Cooper, and Jeff Zheng, Can. J. Chem. 951314 (2017).

${ }^{30}$ J.M. Randazzo, D.M. Mitnik, G. Gasaneo, L.U. Ancarani and F.D. Colavecchia, Eur. Phys. J. D 69, 189 (2015). 
${ }^{31}$ M.J. Ambrosio, F.D. Colavecchia, G. Gasaneo, D.M. Mitnik and L.U.Ancarani, J. Phys. B 48, 055204 (2015).

${ }^{32}$ M.J. Ambrosio, D.M. Mitnik, A. Dorn, L.U. Ancarani and G. Gasaneo, Phys. Rev. A 93, 032705 (2016).

${ }^{33}$ M.J. Ambrosio, D.M. Mitnik, L.U. Ancarani, G. Gasaneo and E.L. Gaggioli, Phys. Rev. A 92, 042704 (2015).

${ }^{34}$ R. Moccia, J. Chem. Phys. 40, 2164 (1964).

${ }^{35}$ M. Dogan, M. Ulu., Z. N. Ozer, M.Yavuz, G. Bozkurt, J. Spectr. 19, 2917 (2013).

${ }^{36}$ M.A. Stevenson, L.R. Hargreaves, B. Lohmann, I. Bray, D.V. Fursa, K. Bartschat, and A. Kheifets, Phys. Rev. A 79, 012709 (2009).

${ }^{37}$ M. A. Haynes and B. Lohmann, Phys. Rev. A 64, 044701 (2001).

${ }^{38}$ C. T. Whelan, Fragmentation Processes:Topics in Atomic and Molecular Physics, C. T. Whelan editor, Cambridge University Press, 223-260 (2013).

${ }^{39}$ M. Stevenson, G.L. Leighton, A. Crowe, K. Bartschat, O.K. Vorov and D.H. Madison, J. Phys. B 38, 433 (2005).

${ }^{40}$ A.J. Murray, B.C.H. Turton and F.H. Read, Rev. Sci. Instrum. 63, 3346 (1992).

${ }^{41}$ A.J. Murray and F.H. Read, Phys. Rev. A 47, 3724 (1993).

${ }^{42}$ A.J. Murray and D. Cvejannovic, J. Phys. B 36, 4875 (2003).

${ }^{43}$ A. Sakaamini, M. Harvey and A.J. Murray (in preparation) 2019. 